\title{
Approximate Solutions to the Discontinuous Riemann-Hilbert Problem of Elliptic Systems of First Order Complex Equations
}

\author{
Guochun Wen ${ }^{1 *}$, Yanhui Zhang ${ }^{2}$, Dechang Chen ${ }^{3 \#}$ \\ ${ }^{1}$ School of Mathematical Sciences, Peking University, Beijing, China \\ ${ }^{2}$ Mathematics Department, Beijing Technology and Business University, Beijing, China \\ ${ }^{3}$ Uniformed Services University of the Health Sciences, Bethesda, USA \\ Email: wengc@math.pku.edu.cn, zhangyanhui@th.btbu.edu.cn, dechang.chen@usuhs.edu
}

Received 23 March 2014; revised 23 April 2014; accepted 30 April 2014

Copyright (C) 2014 by authors and Scientific Research Publishing Inc.

This work is licensed under the Creative Commons Attribution International License (CC BY). http://creativecommons.org/licenses/by/4.0/

(c) (i) Open Access

\begin{abstract}
Several approximate methods have been used to find approximate solutions of elliptic systems of first order equations. One common method is the Newton imbedding approach, i.e. the parameter extension method. In this article, we discuss approximate solutions to discontinuous RiemannHilbert boundary value problems, which have various applications in mechanics and physics. We first formulate the discontinuous Riemann-Hilbert problem for elliptic systems of first order complex equations in multiply connected domains and its modified well-posedness, then use the parameter extensional method to find approximate solutions to the modified boundary value problem for elliptic complex systems of first order equations, and then provide the error estimate of approximate solutions for the discontinuous boundary value problem.
\end{abstract}

\section{Keywords}

Discontinuous Riemann-Hilbert Problem, Elliptic Systems of First Order Complex Equations, Estimates and Existence of Solutions, Multiply Connected Domains

\section{Introduction}

Let $D$ be an $N+1(N \geq 1)$-connected bounded domain in $\mathbb{C}$ with the boundary $\partial D=\Gamma=\bigcup_{j=0}^{N} \Gamma_{j} \in C_{\mu}^{1}(0<\mu<1)$. Without loss of generality, we assume that $D(0 \in D)$ is a circular domain

\footnotetext{
*Deceased.

"I am very grateful for the guidance and help of Professor Guochun Wen, who served as my adviser for many years. 1 will always remember him because he influenced me greatly.
} 
in $|z|<1$, bounded by the $(N+1)$-circles $\Gamma_{j}:\left|z-z_{j}\right|=r_{j}, j=0,1, \cdots, N$ and $\Gamma_{0}=\Gamma_{N+1}:|z|=1$. In this article, the notations are the same as in references [1]-[12]. If the first order elliptic system with $2 n$ unknown real functions

$$
\Phi_{j}\left(x, y, u_{1}, \cdots, u_{2 n}, u_{1 x}, \cdots, u_{2 n x}, u_{1 y}, \cdots, u_{2 n y}\right)=0, j=1, \cdots, 2 n \text { in } D
$$

satisfies certain conditions, then (1.1) can be transformed into the complex form

$$
w_{k \bar{z}}=F_{k}\left(z, w_{1}, \cdots, w_{n}, w_{1 z}, \cdots, w_{n z}\right), k=1, \cdots, n,
$$

where $w_{k}(z)=u_{k}(z)+i u_{k+n}(z), k=1, \cdots, n$ (see Section 4, Chapter 2 in [5]). Its vector form is as follows:

$$
w_{\bar{z}}=F\left(z, w, w_{z}\right), w=\left(w_{1}, \cdots, w_{n}\right)^{\prime}, F=\left(F_{1}, \cdots, F_{n}\right)^{\prime},
$$

where $\left(w_{1}, \cdots, w_{n}\right)^{\prime}=\left(w_{1}, \cdots, w_{n}\right)^{\mathrm{T}}$ is the transposed matrix of $\left(w_{1}, \cdots, w_{n}\right)$. We discuss the first order complex system (1.3) in the form

$$
w_{\bar{z}}=F\left(z, w, w_{z}\right), F=Q^{1} w_{z}+Q^{2} \bar{w}_{\bar{z}}+A^{1} w+A^{2} \bar{w}+A^{3},
$$

in which $Q^{j}=\left(Q_{k m}^{j}\right)$ with $Q_{k m}^{j}=Q_{k m}^{j}\left(z, w, w_{z}\right), A^{j}=\left(A_{k m}^{j}\right)$ with $A_{k m}^{j}=A_{k m}^{j}(z, w), j=1,2$,

$A^{3}=\left(A_{1}^{3}, \cdots, A_{n}^{3}\right)^{\prime}$ with $A_{k}^{3}=A_{k}^{3}(z, w), \quad k, m=1, \cdots, n$.

We assume (1.4) satisfies the following conditions:

Condition C 1) $Q_{k m}^{j}(z, w, U), A_{k m}^{j}(z, w), A_{k}^{3}(z, w)(j=1,2, k, m=1, \ldots, n)$ are continuous in $w \in \mathbb{C}^{n}$ for almost every point $z \in D, U \in \mathbb{C}^{n}$.

2) The above functions are measurable in $z \in D$ for all systems of continuous functions $w(z)$ in $D^{*}=\bar{D} \backslash Z$ and any systems of measurable functions $U(z)$ in $D^{*}$ and satisfy

$$
\begin{array}{r}
L_{p}\left[A_{k m}^{j}(z, w), \bar{D}\right] \leq k_{0}, 1 \leq j \leq 2, \quad L_{p}\left[A_{k}^{3}(z, w), \bar{D}\right] \leq k_{2}, 1 \leq k, m \leq n, \\
L_{p}\left[A_{k m}^{j}(z, w), \bar{D}\right] \leq k_{1}, 1 \leq j \leq 2,1 \leq k<m \leq n,
\end{array}
$$

where $Z$ is as stated in (1.8) below, $p(>2)$ and $k_{j}(j=0,1,2)$ are non-negative constants.

3) The complex system (1.4) satisfies the following ellipticity condition

$$
\left\{\begin{array}{l}
\left|F_{k}\left(z, w^{1}, U^{1}\right)-F_{k}\left(z, w^{2}, U^{2}\right)\right| \leq \sum_{m=1}^{n}\left[q_{k m}\left|U_{m}^{1}-U_{m}^{2}\right|+\sum_{m=1}^{n} \kappa_{k m}\left|w_{m}^{1}-w_{m}^{2}\right|\right], \\
\sum_{k=1}^{n} \sum_{m=1}^{n} q_{k m}=\sum_{k=1}^{n} q_{k} \leq q_{0}<1, q_{k m} \leq k_{3} \leq k_{0}, 1 \leq k<m \leq n, \\
\sum_{k=1}^{n} \sum_{m=1}^{n} \kappa_{k m}=\sum_{k=1}^{n} \kappa_{k} \leq k_{0}, \kappa_{k m} \leq k_{3} \leq k_{0}, 1 \leq k<m \leq n,
\end{array}\right.
$$

where $q_{k m}, q_{k}, \kappa_{k m}, \kappa_{k}(k, m=1, \cdots, n), q_{0}, k_{0}, k_{3}$ are non-negative constants.

For convenience, $R(z) w(z) \in C_{\beta}(\bar{D})$ and $R(z) \tilde{R}(z) U(z) \in L_{p_{0}}(\bar{D})$ are used to indicate

$R(z) w_{k}(z) \in C_{\beta}(\bar{D})$ and $R(z) \tilde{R}(z) U_{k}(z) \in L_{p_{0}}(\bar{D})$, respectively, $k=1, \cdots, n$, and we define the following:

$$
C_{\beta}[R w, \bar{D}]=\sum_{k=1}^{n} C_{\beta}\left[R w_{k}, \bar{D}\right], \quad L_{p_{0}}[R \tilde{R} U, \bar{D}]=\sum_{k=1}^{n} L_{p_{0}}\left[R \tilde{R} U_{k}, \bar{D}\right]
$$

in which $w=\left(w_{1}, \cdots, w_{n}\right)^{\prime}, U=\left(U_{1}, \cdots, U_{n}\right)^{\prime}$, and $R(Z), \tilde{R}(Z)$ are stated as in (1.12), (2.1) below, and $\beta(0<\beta<1)$ and $p_{0}\left(2<p_{0} \leq p\right)$ are non-negative constants.

The so-called Riemann-Hilbert boundary value problem for the complex system (1.4) may be formulated as follows.

Problem A Find a system of continuous solutions $w(z)=\left(w_{1}(z), \cdots, w_{n}(z)\right)^{\prime}$ in $D^{*}=\bar{D} \backslash Z$ of (1.4), which satisfies the boundary condition 


$$
\operatorname{Re}[\overline{\lambda(z)} w(z)]=r(z), z \in \Gamma^{*}=\Gamma \backslash Z,
$$

in which $\lambda(z)=\left(\lambda_{k m}(z)\right)$ with $\left|\lambda_{k m}\right|=1$ for $z \in \Gamma, 1 \leq k, m \leq n, \quad r(z)=\left(r_{1}(z), \cdots, r_{n}(z)\right)^{\prime}$, and $Z=\left\{t_{1}, t_{2}, \cdots, t_{m^{\prime}}\right\}$ are the first kind of discontinuous points of $\lambda_{k m}(z)$ on $\Gamma$.

Denote by $\lambda_{k m}\left(t_{j}-0\right)$ and $\lambda_{k m}\left(t_{j}+0\right)$ the left limit and right limit of $\lambda_{k m}(z)$ as $z \rightarrow t_{j}\left(j=1,2, \cdots, m^{\prime}, k, m=1, \cdots, n\right)$ on $\Gamma$, and

$$
\begin{gathered}
\mathrm{e}^{i \phi_{k m j}}=\frac{\lambda_{k m}\left(t_{j}-0\right)}{\lambda_{k m}\left(t_{j}+0\right)}, \gamma_{k m j}=\frac{1}{\pi i} \ln \left[\frac{\lambda_{k m}\left(t_{j}-0\right)}{\lambda_{k m}\left(t_{j}+0\right)}\right]=\frac{\phi_{k m j}}{\pi}-K_{k m j}, \\
K_{k m j}=\left[\frac{\phi_{k m j}}{\pi}\right]+J_{k m j}, J_{k m j}=0 \text { or } 1, j=1, \cdots, m^{\prime}, 1 \leq k, m \leq n,
\end{gathered}
$$

where $0 \leq \gamma_{k m j}<1$ when $J_{k m j}=0$, and $-1<\gamma_{k m j}<0$ when $J_{k m j}=1, k, m=1, \cdots, n, j=1, \cdots, m^{\prime}$. There is no harm in assuming that the partial indexes $K_{k}$ of $\lambda(z)$ on $\Gamma_{k}\left(k=1, \cdots, N_{0}(\leq N)\right)$ are not integers, and the partial indexes $K_{k}$ of $\lambda(z)$ on $\Gamma_{k}\left(j=0, N_{0}+1, \cdots, N\right)$ are integers. Set

$$
K_{k}=\frac{1}{2 \pi} \Delta_{\Gamma} \arg \lambda_{k k}(z)=\sum_{j=1}^{m^{\prime}} \frac{K_{k k j}}{2}, k=1, \cdots, n,
$$

and we call $K=\left(K_{1}, \cdots, K_{n}\right)^{\prime}$ the index of Problem A.

For problem A, we will assume $\lambda_{k m}(z), r_{k}(z)(k, m=1, \cdots, n)$ satisfy the conditions

$$
\begin{gathered}
C_{\alpha}\left[\lambda_{k k j}(z), \hat{\Gamma}_{j}\right] \leq k_{0}, k=1, \cdots, n, C_{\alpha}\left[\lambda_{k m j}, \hat{\Gamma}_{j}\right] \leq k_{4}, 1 \leq k<m \leq n, \\
C_{\alpha}\left[\left|z-t_{j}\right|^{\beta_{k j j}}\left|z-t_{j-1}\right|^{\beta_{k k j-1}} r_{k}(z), \hat{\Gamma}_{j}\right] \leq k_{5}, 1 \leq k \leq n, j=1, \cdots, m^{\prime},
\end{gathered}
$$

in which $\Gamma_{j}$ is an open arc from the point $t_{j-1}$ to $t_{j}$ on $\Gamma \alpha(1 / 2<\alpha<1), k_{4}, k_{5}$ are non-negative constants, $\quad \beta_{k m j}+\left|\gamma_{k m j}\right|<1, j=1, \cdots, m^{\prime}, k, m=1, \cdots, n$. Moreover, we require that the solution $w(z)$ possess the property

$$
\begin{aligned}
& R(z) w(z)=C_{\delta}(\bar{D}), R(z)=\Pi_{j=1}^{m^{\prime}}\left|z-t_{j}\right|^{-\eta_{j} / \tau^{2}}, \eta_{j}=\max \left(\eta_{11 j}, \eta_{12 j}, \cdots, \eta_{n-1, n j}, \eta_{n, n j}\right), j=1, \cdots, m^{\prime}, \\
& \eta_{k m j}=\left\{\begin{array}{l}
\beta_{k m j}+\tau, \text { for } \gamma_{k m j} \geq 0, \text { and } \gamma_{k m j}<0, \beta_{k m j} \geq\left|\gamma_{k m j}\right|, \\
\left|\gamma_{k m j}\right|+\tau, \text { for } \gamma_{k m j}<0, \beta_{k m j}<\left|\gamma_{k m j}\right|, j=1, \cdots, m^{\prime},
\end{array}\right.
\end{aligned}
$$

in $D^{*}$, where $|R(z)| \leq 1$ in $\bar{D}$, and $\delta, \tau\left(<\min \left(\alpha, 1-2 / p_{0}\right)\right)$ are small positive constants.

In general, Problem A may not be solvable. Hence we propose a modified problem as follows.

Problem B Find a system of continuous solutions $w(z)$ of the complex equation (1.4) in $D^{*}$, which satisfies the modified boundary condition

$$
\operatorname{Re}[\overline{\lambda(z)} w(z)]=r(z)=h(z), \quad z \in \Gamma^{*}=\Gamma \backslash Z
$$

Here

$$
\left.\frac{h_{k}(z) \lambda_{k}(z)}{X_{k}(z)}=\left\{\begin{array}{l}
0, \quad z \in \Gamma_{0}, \\
h_{k j}, z \in \Gamma_{j}, j=1, \cdots, N,
\end{array}\right\} \quad \begin{array}{l}
\text { if } K_{k} \geq 0, \\
h_{k j}, z \in \Gamma_{j}, j=1, \cdots, N, \\
{\left[1+(-1)^{2 K_{k}}\right] h_{k 0}+\operatorname{Re}^{\left[K_{k} \mid+1 / 2\right]-1}\left(h_{k m}^{+}+i h_{k m}^{-}\right) z^{m}, z \in \Gamma_{0},}
\end{array}\right\} \quad k=1, \cdots, n,(1.14)
$$

in which $h_{k j}\left(j=\left[1-(-1)^{2 K_{k}}\right] / 2, \cdots, N\right), \quad h_{k m}^{+}, h_{k m}^{-}\left(m=1, \cdots,\left[\left|K_{k}\right|+1 / 2\right]-1, k=1, \cdots, n\right)$ are unknown real 
constants to be determined appropriately, and $h_{k, N+1}\left(=h_{k 0}\right)=0$, if $2\left|K_{k}\right|$ is an odd integer. More description on $\lambda_{k}(z)$ and $X_{k}(z)$ are given below. We begin with the following function

$$
\begin{aligned}
& Y_{k}(z)=\prod_{j=1}^{m_{0}}\left(z-t_{j}\right)^{\gamma_{k, m_{j}}} \prod_{l=l}^{N}\left(z-z_{l}\right)^{-\left[\tilde{K}_{k l}\right]} \prod_{j=m_{0}+1}^{m_{1}}\left(\frac{z-t_{j}}{z-z_{1}}\right)^{\gamma_{k, m_{j}}}\left(\frac{z-t_{1}^{\prime}}{z-z_{1}}\right) \\
& \times \prod_{j=m_{N_{0}-1}+1}^{m_{N_{0}}}\left(\frac{z-t_{j}}{z-z_{N_{0}}}\right)^{\gamma_{k, m_{j}}}\left(\frac{z-t_{N_{0}}^{\prime}}{z-z_{N_{0}}}\right)_{j=m_{N_{0}+1}}^{m_{N_{0}+1}}\left(\frac{z-t_{j}}{z-z_{N_{0}+1}}\right)^{\gamma_{k, m_{j}}} \cdots \prod_{j=m_{N-1}+1}^{m}\left(\frac{z-t_{j}}{z-z_{N}}\right)^{\gamma_{k, m_{j}}},
\end{aligned}
$$

where $\tilde{K}_{k l}=\sum_{j=m_{l-1}+1}^{m_{l}} K_{k j}$ denotes the partial index on $\Gamma_{l}(l=1, \cdots, N), t_{l}^{\prime}\left(\in \Gamma_{l}, l=1, \cdots, N_{0}\right)$ are fixed points, which are not the discontinuous points from $Z$. Note that the positive direction applies to the boundary circles $\Gamma_{j}(j=0,1, \cdots, N)$. Similarly to (1.7)-(1.12), Chapter V, [2], we see that

$$
\frac{\lambda_{k k}\left(t_{j}-0\right)}{\lambda_{k k}\left(t_{j}+0\right)} \overline{\left[\frac{Y_{k}\left(t_{j}-0\right)}{Y_{k}\left(t_{j}+0\right)}\right]}=\frac{\lambda_{k k}\left(t_{j}-0\right)}{\lambda_{k k}\left(t_{j}+0\right)} \mathrm{e}^{-i \pi \gamma_{k j}}= \pm 1, k, j=1, \cdots, m .
$$

Clearly, with certain modification on the symbols on some arcs on $\Gamma, \lambda_{k k}(z) \overline{Y_{k}(z)} /\left|Y_{k}(z)\right|$ on $\Gamma$ is seen to be continuous. In this case, its index

$$
\kappa_{k}=\frac{1}{2 \pi} \Delta_{\Gamma}\left[\lambda_{k k}(z) \overline{Y_{k}(z)}\right]=K_{k}-\frac{N_{0}}{2}, k=1, \cdots, n
$$

are integers. And we have the following:

$$
\begin{gathered}
X_{k}(z)=\left\{\begin{array}{l}
i z^{\left[\kappa_{k}\right]} \mathrm{e}^{i S_{k}(z)} Y_{k}(z), \quad z \in \Gamma_{0}, \\
i \mathrm{e}^{i \theta_{k j}} \mathrm{e}^{i S_{k}(z)} Y_{k}(z), z \in \Gamma_{j}, j=1, \cdots, N,
\end{array} \quad \operatorname{Im}\left[\overline{\lambda_{k}(z)} X_{k}(z)\right]=0, z \in \Gamma, k=1, \cdots, n,\right. \\
\operatorname{Re} S_{k}(z)=\left\{\begin{array}{l}
\arg \lambda_{k k}(z)-\left[\kappa_{k}\right] \arg z-\arg Y_{k}(z), z \in \Gamma_{0}, \\
\arg \lambda_{k k}(z)-\arg Y_{k}(z)-\theta_{k j}, \quad z \in \Gamma_{j}, j=1, \cdots, N,
\end{array}, \operatorname{Im}\left[S_{k}(1)\right]=0, k=1, \cdots, n,\right.
\end{gathered}
$$

in which $S_{k}(z)(k=1, \cdots, n)$ are solutions of the modified Dirichlet problems with the above boundary conditions for analytic functions, $\theta_{k j}(j=1, \cdots, N, k=1, \cdots, n)$ are real constants, and $\kappa_{k}=K_{k}-N_{0} / 2(k=1, \cdots, n)$.

In addition, we may assume that the solution $w(z)$ satisfies the following point conditions

$$
\operatorname{Im}\left[\overline{\lambda\left(a_{j}\right)} w\left(a_{j}\right)\right]=b_{k j}, j \in J_{k}=\left\{1, \cdots, 2 K_{k}+1\right\}, \text { if } K_{k} \geq 0, k=1, \cdots, n,
$$

where $a_{j} \in \Gamma_{0}\left(j \in J_{k}\right)$ are distinct points, and $b_{k j}\left(j \in J_{k}, k=1, \cdots, n\right)$ are all real constants satisfying the conditions

$$
\left|b_{k j}\right| \leq k_{6}, j \in J_{k}, k=1, \cdots, n,
$$

for a positive constant $k_{6}$. Problem B with $A_{3}(z, w)=0$ in $D, c(z)=0$ on $\Gamma$ and $b_{k j}=0\left(j \in J_{k}, k=1, \cdots, n\right)$ is called Problem $\mathrm{B}_{0}$.

If $\lambda_{k k}(z)=1, \lambda_{k m}=0, k \neq m=1, \cdots, n$, then Problem B for (1.4) is the modified Dirichlet boundary value problem for (1.4). It is easy to see that the solutions of (1.4) include the generalized hyperanalytic functions as special cases. In fact, if (1.4) is linear, and $Q_{k m}^{2}=0,1 \leq k, m \leq n, \quad Q_{k m}^{1}=A_{k m}^{1}=A_{k m}^{2}=0,1 \leq k<m \leq n$ and $Q_{k+1, k}^{1}=Q_{k+2, k+1}^{1}, \quad A_{k+1, k}^{j}=A_{k+2, k+1}^{j}, 1 \leq k \leq N-1$, then the solutions of (1.4) are called generalized hyperanalytic functions.

\section{Parameter Extension Method of the Discontinuous Riemann-Hilbert Problem for Elliptic Systems of First Order Complex Equations}

We begin with the following estimates of the solution for problem B.

Theorem 2.1 Suppose that the complex system (1.4) satisfies Condition $\mathrm{C}$ and the constants $k_{1}, k_{3}, k_{4}$ in 
(1.6), (1.7), (1.11) are small enough. Then any solution $w(z)=\left(w_{1}(z), \cdots, w_{n}(z)\right)^{\prime}$ of Problem B for (1.4) satisfies the estimate

$$
\begin{aligned}
& C_{\beta}[R(z) w(z), \bar{D}]+L_{p_{0}}\left[R(z) \tilde{R}(z)\left(\left|w_{\bar{z}}\right|+\left|w_{z}\right|\right), \bar{D}\right] \\
& =\sum_{k=1}^{n}\left\{C_{\beta}\left[R(z) w_{k}(z), \bar{D}\right]+L_{p_{0}}\left[R(z) \tilde{R}(z)\left(\left|w_{k \bar{z}}\right|+\left|w_{k z}\right|\right), \bar{D}\right]\right\} \leq M_{1} k_{*},
\end{aligned}
$$

where $\tilde{R}(z)=\Pi_{j=1}^{m^{\prime}}\left|z-t_{j}\right|^{1 / \tau^{2}}$ with $|\tilde{R}(z)| \leq 1$ in $\bar{D}, \beta=\min \left(\alpha, 1-2 / p_{0}\right), p_{0}\left(2<p_{0} \leq p\right)$,

$k_{*}=k_{2}+k_{5}+k_{6}, \quad M_{1}=M_{1}\left(q_{0}, p_{0}, k_{0}, \beta, K, D\right)$ with $K=\left(K_{1}, \cdots, K_{n}\right)^{\prime}$ are non-negative constants.

Proof There is no harm in assuming that $k_{*}=k_{2}+k_{5}+k_{6} \neq 0$. Let $W(z)=w(z) / k_{*}$. It can be seen that $W(z)$ is a solution of the following boundary value problem

$$
\begin{aligned}
& W_{\bar{z}}= Q^{1} W_{z}+Q^{2} \overline{W_{z}}+A^{1} W+A^{2} \bar{W}+A^{3} / k_{*}, z \in D, \\
& \operatorname{Re}[\overline{\lambda(z)} W(z)]=[r(z)+h(z)] / k_{*}, \quad z \in \Gamma, \\
& \operatorname{Im}\left[\overline{\lambda\left(a_{j}\right)} W\left(a_{j}\right)\right]=b_{j} / k_{*}, j \in J_{k}, k=1, \cdots, n,
\end{aligned}
$$

in which

$$
L_{p}\left[A_{k}^{3} / k_{*}, \bar{D}\right] \leq 1, C_{\alpha}\left[r_{k}(z) / k_{*}, \Gamma\right] \leq 1,\left|b_{k j} / k_{*}\right| \leq 1, j \in J_{k}, k=1, \cdots, n .
$$

Following the proof of the Theorem 2.1 of Chapter VI in [1], we can derive the estimate

$$
\begin{aligned}
& C_{\beta}[R(z) W(z), \bar{D}]+L_{p_{0}}\left[R(z) \tilde{R}(z)\left(\left|W_{\bar{z}}\right|+\left|W_{z}\right|\right) \bar{D}\right] \leq M_{1} \\
& =M_{1}\left(q_{0}, p_{0}, k_{0}, \beta, K, D\right) .
\end{aligned}
$$

From the above estimate, it immediately follows that the estimate (2.1) is true.

In addition, we assume that (1.4) satisfies the following condition: For any continuous vectors $w^{1}(z), w^{2}(z)$ and any measurable vector $U(z) \in L_{p_{0}}(\bar{D})$,

$$
\begin{aligned}
& F\left(z, w^{1}, U\right)-F\left(z, w^{2}, U\right) \\
& =\tilde{Q}\left(z, w^{1}, w^{2}, U\right) U+\tilde{A}\left(z, w^{1}, w^{2}, U\right)\left(w^{1}-w^{2}\right),
\end{aligned}
$$

where $\tilde{Q}=\left(\tilde{Q}_{k m}\right), \tilde{A}=\left(\tilde{A}_{k m}\right)$ satisfy the condition

$$
\begin{gathered}
\left|\tilde{Q}_{k m}\right| \leq q_{k m}, \sum_{k=1}^{n} \sum_{m=1}^{n} q_{k m}=\sum_{k=1}^{n} q_{k} \leq q_{0}<1, q_{k m} \leq k_{3} \leq k_{0}, 1 \leq k<m \leq n, \lim _{x \rightarrow \infty} \\
L_{p_{0}}\left[\tilde{A}_{k m}, \bar{D}\right] \leq k_{0}, 1 \leq k, m \leq n, L_{p_{0}}\left[\tilde{A}_{k m}, \bar{D}\right] \leq k_{1} \leq k_{0}, 1 \leq k<m \leq n,
\end{gathered}
$$

in which $p_{0}\left(2 \leq p_{0} \leq p\right), k_{0}, k_{1}$ are non-negative constants.

Now, we prove that there exists a unique solution of the modified Riemann-Hilbert problem (Problem B) for analytic vectors by the parameter extensional method.

Theorem 2.2 Let $k_{4}$ in (1.11) be a sufficiently small positive constant. Then Problem B for analytic vectors has a solution.

Proof We consider the modified Riemann-Hilbert problem (Problem $\mathrm{B}^{\prime}$ ) for analytic vectors with the boundary conditions

$$
\begin{array}{r}
\operatorname{Re}[\overline{\Lambda(\zeta)} w(z)]+t \operatorname{Re}[\overline{\Delta(z)} w(z)]=\hat{r}(z)+h(z), \quad z \in \Gamma, \\
\operatorname{Im}\left[\overline{\Lambda\left(a_{j}\right)} w\left(a_{j}\right)\right]+t \operatorname{Im}\left[\overline{\Delta\left(a_{j}\right)} w\left(a_{j}\right)\right]=B_{j}, \quad j \in J_{k}, 1 \leq k \leq n,
\end{array}
$$

where 


$$
\begin{aligned}
& \Lambda(z)=\left(\Lambda_{k m}(z)\right), \Lambda_{k m}(z)=\left\{\begin{array}{ll}
\lambda_{k m}(z), & k \geq m, \\
0, & k<m,
\end{array} \text { on } \Gamma,\right. \\
& \Delta(z)=\left(\Delta_{k m}(z)\right), \Delta_{k m}(z)=\left\{\begin{array}{ll}
0, & k \geq m, \\
\lambda_{k m}(z), & k<m,
\end{array} \text { on } \Gamma,\right.
\end{aligned}
$$

in which $t(0 \leq t \leq 1)$ is a real parameter, and $\hat{r}(z)=\left(\hat{r}_{1}(z), \cdots, \hat{r}_{n}(z)\right)^{\prime}$ is any vector of real functions, $\left|z-t_{j-1}\right|^{\beta_{k(j-1)-1}}\left|z-t_{j}\right|^{\beta_{k j-1}} \hat{r}_{k}(z) \in C_{\alpha}\left(\Gamma_{j}\right), 0<\alpha<1, j=1, \cdots, m^{\prime}, k=1, \cdots, n$, and $B_{j}=\left(B_{j 1}, \cdots, B_{j n}\right)^{\prime}$ is any

vector of constants. When $t=0$, it is clear that Problem $\mathrm{B}^{\prime}$ for analytic vectors has a unique solution (see [1]). If Problem $\mathrm{B}^{\prime}$ with $t=t_{0}\left(0 \leq t_{0}<1\right)$ for analytic vectors is solvable, we shall prove that there exists a positive number $\delta$ independent of $t_{0}$, such that Problem $\mathrm{B}^{\prime}$ for every $t \in E=\left\{\left|t-t_{0}\right| \leq \delta, 0 \leq t \leq 1, \delta>0\right\}$ has a unique solution. In fact, the boundary conditions (2.9), (2.10) can be rewritten in the form

$$
\begin{aligned}
& \operatorname{Re}[\overline{\Lambda(z)} w(z)]+t_{0} \operatorname{Re}[\overline{\Delta(z)} w(z)] \\
&=\left(t_{0}-t\right) \operatorname{Re}[\overline{\Delta(z)} w(z)]+\hat{r}(z)+h(z), \quad z \in \Gamma, \\
& \operatorname{Im}\left[\overline{\Lambda\left(a_{j}\right)} w\left(a_{j}\right)\right]+t_{0} \operatorname{Im}\left[\overline{\Delta\left(a_{j}\right)} w\left(a_{j}\right)\right] \\
&=\left(t_{0}-t\right) \operatorname{Im}\left[\overline{\Delta\left(a_{j}\right)} w\left(a_{j}\right)\right]+B_{j}, j \in J_{k}, 1 \leq k \leq n .
\end{aligned}
$$

Substituting the zero vector $w^{0}(z)=(0, \cdots, 0)^{\prime}$ into the position of $w(z)$ on the right hand side of (2.11) and (2.12), by the hypothesis, the boundary value problem (2.11), (2.12) for analytic vectors has a unique solution $w^{1}(z)=\left(w_{1}^{1}(z), \cdots, w_{n}^{1}(z)\right)$ and $R(z) w_{k}^{1}(z) \in C_{\alpha}(\bar{D}), k=1, \cdots, n$. Using the successive iteration, we can find a sequence $\left\{w^{n}(z)\right\}$ of analytic vectors, which satisfies the boundary conditions

$$
\begin{aligned}
& \operatorname{Re}\left[\overline{\Lambda(z)} w^{n+1}(z)\right]+t_{0} \operatorname{Re}\left[\overline{\Delta(z)} w^{n+1}(z)\right] \\
&=\left(t_{0}-t\right) \operatorname{Re}\left[\overline{\Delta(z)} w^{n}(z)\right]+\hat{r}(z)+h(z), \quad z \in \Gamma, \\
& \operatorname{Im}\left[\overline{\Lambda\left(a_{j}\right)} w^{n+1}\left(a_{j}\right)\right]+t_{0} \operatorname{Im}\left[\overline{\Delta\left(a_{j}\right)} w^{n+1}\left(a_{j}\right)\right] \\
&=\left(t_{0}-t\right) \operatorname{Im}\left[\overline{\Delta\left(a_{j}\right)} w^{n}\left(a_{j}\right)\right]+B_{j}, \quad j \in J_{k}, 1 \leq k \leq n .
\end{aligned}
$$

From (2.13) and (2.14), we have

$$
\begin{gathered}
\operatorname{Re}\left[\overline{\Lambda(z)}\left(w^{n+1}-w^{n}\right)\right]+t_{0} \operatorname{Re}\left[\overline{\Delta(z)}\left(w^{n+1}-w^{n}\right)\right] \\
=\left(t_{0}-t\right) \operatorname{Re}\left[\overline{\Delta(z)}\left(w^{n}-w^{n-1}\right)\right]+h(z), \quad z \in \Gamma, \\
\left.\left\{\operatorname{Im}\left[\overline{\Lambda(z)}\left(w^{n+1}-w^{n}\right)\right]+t_{0} \operatorname{Im}\left[\overline{\Delta(z)}\left(w^{n+1}-w^{n}\right)\right]\right\}\right|_{z=a_{j}} \\
=\left.\left(t_{0}-t\right) \operatorname{Im}\left[\overline{\Delta(z)}\left(w^{n}-w^{n-1}\right)\right]\right|_{z=a_{j}}+B_{j}, \quad j \in J_{k}, 1 \leq k \leq n, n=1,2, \cdots
\end{gathered}
$$

In accordance with Theorem 2.1, we can conclude

$$
\begin{aligned}
C_{\alpha}\left[R\left(w^{n+1}-w^{n}\right), \bar{D}\right] & \leq M_{2} C_{\alpha}\left[\left(t-t_{0}\right) \overline{\Delta(z)} R\left(w_{n}-w_{n-1}\right), \Gamma\right] \\
& \leq M_{2}\left|t-t_{0}\right| k_{0} C_{\alpha}\left[R\left(w^{n}-w^{n-1}\right), \Gamma\right] \\
& \leq M_{2} k_{0}\left|t-t_{0}\right| C_{\alpha}\left[R\left(w^{n}-w^{n-1}\right), \bar{D}\right],
\end{aligned}
$$


where $M_{2}=M_{2}\left(k^{\prime}, \beta, K, D\right)$ with $k^{\prime}=\left(k_{0}, k_{1}, \cdots, k_{4}\right)$, and $K=\left(K_{1}, \cdots, K_{n}\right)^{\prime}$. Choosing a positive constant $\delta$, such that $\delta M_{2} k_{0}<1$, it is not difficult to see that

$$
C_{\alpha}\left[R\left(w^{n+1}-w^{n}\right), \bar{D}\right] \leq \frac{1}{2} C_{\alpha}\left[R\left(w^{n}-w^{n-1}\right), \bar{D}\right] \leq \frac{1}{2^{N}} C_{\alpha}\left[R w^{1}, \bar{D}\right],
$$

and

$$
C_{\alpha}\left[R\left(w^{n}-w^{m}\right), \bar{D}\right] \leq \frac{1}{2^{N}} C_{\alpha}\left[R w^{1}, \bar{D}\right]
$$

for $n \geq m>N$, where $N$ is a positive integer. This shows that

$$
C_{\alpha}\left(R\left(w^{n}-w^{m}\right)\right) \rightarrow 0 \text { as } n, m \rightarrow \infty .
$$

Hence, there exists an analytic vector $w^{*}(z)=\left(w_{1}^{*}(z), \cdots, w_{n}^{*}(z)\right)$, such that

$$
C_{\alpha}\left[R\left(w^{n}-w^{*}\right), \bar{D}\right] \rightarrow 0 \text { as } n \rightarrow \infty \text {. }
$$

Thus $w_{*}(z)$ is a solution of Problem $\mathrm{B}^{\prime}$ with $t \in E$. From this we can derive that Problem $\mathrm{B}^{\prime}$ with $t=1, R(z)=r(z) 3011, B_{j}=b_{j}\left(j \in J_{k}, 1 \leq k \leq n\right)$, i.e. Problem B for analytic vectors is solvable.

Next we prove the solvability of Problem B for the system (1.4).

Theorem 2.3 Let the nonlinear elliptic system (1.4) satisfy Condition C, and $k_{1}, k_{3}, k_{4}$ in (1.6), (1.7), (1.11) be sufficiently small positive constants. Then Problem B for the complex system (1.4) is solvable.

Proof We consider the nonlinear elliptic complex system with the parameter $t \in[0,1]$ :

$$
w_{\bar{z}}-t F\left(z, w, w_{z}\right)=A(z),
$$

where $A(z)=\left(A_{1}(z), \cdots, A_{n}(z)\right)^{\prime}$ is any measurable vector in $D$ and $R(z) \tilde{R}(z) A_{j}(z) \in L_{p_{0}}(\bar{D}), j=1, \cdots, n$. Applying Theorem 2.2, we see that Problem B for (2.19) with $t=0$ is solvable, and the solution $w(z)$ can be expressed as

$$
w(z)=w_{0}(z)+\Psi(z), \Psi(z)=T A-\frac{1}{\pi} \iint_{D} \frac{A(\zeta)}{\zeta-z} \mathrm{~d} \sigma_{\zeta},
$$

where $w_{0}(z)$ is an analytic vector satisfying the boundary conditions

$$
\begin{array}{r}
\operatorname{Re}\left[\overline{\lambda(z)}\left(w_{0}(z)+\Psi(z)\right)\right]=r(z)+h(z), \quad z \in \Gamma, \\
\operatorname{Im}\left[\overline{\lambda\left(a_{j}\right)}\left(w_{0}\left(a_{j}\right)+\Psi\left(a_{j}\right)\right)\right]=b_{j}, \quad j \in J_{k}, 1 \leq k \leq n .
\end{array}
$$

Suppose that when $t=t_{0}\left(0 \leq t_{0}<1\right)$, Problem B for the system (2.19) has a unique solution. Then we shall prove that there exists a neighborhood of $t_{0}: E=\left\{\left|t-t_{0}\right| \leq \delta, 0 \leq t \leq 1, \delta>0\right\}$, so that for every $t \in E$ and any function $R(z) \tilde{R}(z) A(z) \in L_{p_{0}}(\bar{D})$, Problem B for (2.19) is solvable. In fact, the complex system (2.19) can be written in the form

$$
w_{\bar{z}}-t_{0} F\left(z, w, w_{z}\right)=\left(t-t_{0}\right) F\left(z, w, w_{z}\right)+A(z) .
$$

Suppose that Problem B for (2.13) with $t=t_{0}\left(0 \leq t_{0}<1\right)$ is solvable, by using the similar method as in the proof of Theorem 2.2, we can find a positive constant $\delta$, so that for every $t \in E=\left\{\left|t-t_{0}\right| \leq \delta, 0 \leq t \leq 1\right\}$, there exists a sequence $\left\{w^{n}(z)\right\}$ of solutions satisfying

$$
w_{n+1 \bar{z}}-t_{0} F\left(z, w_{n+1}, w_{n+1 z}\right)=\left(t-t_{0}\right) F\left(z, w_{n}, w_{n z}\right)+A(z), n=1,2, \cdots .
$$

The difference of the above equations for $n+1$ and $n$ is as follows:

$$
\begin{aligned}
& \left(w_{n+1}-w_{n}\right)_{\bar{z}}-t_{0}\left[F\left(z, w_{n+1}, w_{n+1 z}\right)-F\left(z, w_{n}, w_{n z}\right)\right] \\
& =\left(t-t_{0}\right)\left[F\left(z, w_{n}, w_{n z}\right)-F\left(z, w_{n-1}, w_{n-1 z}\right)\right], n=1,2, \cdots .
\end{aligned}
$$


From Condition $\mathrm{C}$, we can derive that

$$
\begin{aligned}
& F\left(z, w_{n+1}, w_{n+1 z}\right)-F\left(z, w_{n}, w_{n z}\right) \\
& =F\left(z, w_{n+1}, w_{n+1 z}\right)-F\left(z, w_{n+1}, w_{n z}\right)+F\left(z, w_{n+1}, w_{n z}\right)-F\left(z, w_{n}, w_{n z}\right) \\
& =\tilde{Q}_{n+1}(z)\left(w_{n+1}-w_{n}\right)_{z}+\tilde{A}_{n+1}(z)\left(w_{n+1}-w_{n}\right), \\
& \left|\tilde{Q}_{n+1}(z)\right| \leq q_{0}<1, \tilde{A}_{n+1}(z) \in L_{p_{0}}(\bar{D}), n=1,2, \cdots,
\end{aligned}
$$

and

$$
\begin{aligned}
& L_{p_{0}}\left[R \tilde{R}\left(F\left(z, w_{n}, w_{n z}\right)-F\left(z, w_{n-1}, w_{n-1 z}\right)\right), \bar{D}\right] \\
& \leq q_{0} L_{p_{0}}\left[R \tilde{R}\left(w_{n}-w_{n-1}\right)_{z}, \bar{D}\right]+n k_{0} C\left[R\left(w_{n}-w_{n-1}\right), \bar{D}\right] \\
& \leq\left(q_{0}+n k_{0}\right)\left[C_{\beta}\left[R\left(w_{n}-w_{n-1}\right), \bar{D}\right]+L_{p_{0}}\left[R \tilde{R}\left(\left|\left(w_{n}-w_{n-1}\right)_{\bar{z}}\right|+\left|\left(w_{n}-w_{n-1}\right)_{z}\right|\right), \bar{D}\right]\right] \\
& =\left(q_{0}+n k_{0}\right) L_{n} .
\end{aligned}
$$

Moreover, $w_{n+1}(z)-w_{n}(z)$ satisfies the homogeneous boundary conditions

$$
\begin{array}{r}
\operatorname{Re}\left[\overline{\lambda(z)}\left[w_{n+1}(z)-w_{n}(z)\right]\right]=h(z), \quad z \in \Gamma, \\
\operatorname{Im}\left[\overline{\lambda\left(a_{j}\right)}\left(w_{n+1}\left(a_{j}\right)-w_{n}\left(a_{j}\right)\right)\right]=0, j \in J_{k}, 1 \leq k \leq n .
\end{array}
$$

Similarly to Theorem 3.3, Chapter I, [1], we have

$$
\begin{aligned}
L_{n+1} & =C_{\beta}\left[R\left(w_{n+1}-w_{n}\right), \bar{D}\right]+L_{p_{0}}\left[R \tilde{R}\left(\left|\left(w_{n+1}-w_{n}\right)_{z}\right|+\left|\left(w_{n+1}-w_{n}\right)_{z}\right|\right), \bar{D}\right] \\
& \leq M_{3}\left|t-t_{0}\right|\left(q_{0}+n k_{0}\right) L_{n},
\end{aligned}
$$

where $M_{3}=M_{3}\left(q_{0}, p_{0}, k^{\prime}, \alpha, K, D\right)\left(k^{\prime}=\left(k_{0}, k_{2}, k_{5}, k_{6}\right)\right)$ are positive constants. Provided $\delta(>0)$ is small enough, so that $\eta=\delta M_{3}\left(q_{0}+n k_{0}\right)<1$, we can obtain

$$
L_{n+1} \leq \eta L_{n} \leq \eta^{n} L_{1}=\eta^{n}\left[C_{\beta}\left(R w_{1}, \bar{D}\right)+L_{p_{0}}\left(R \tilde{R}\left(\left|w_{1 \bar{z}}\right|+\left|w_{1 z}\right|\right), \bar{D}\right)\right]
$$

for every $t \in E$. Thus

$$
\begin{aligned}
S\left(w_{n}-w_{m}\right) & =C_{\beta}\left[R\left(w_{n}-w_{m}\right), \bar{D}\right]+L_{p_{0}}\left[R \tilde{R}\left(\left|\left(w_{n}-w_{m}\right)_{\bar{z}}\right|+\left|\left(w_{n}-w_{m}\right)_{z}\right|\right), \bar{D}\right] \\
& \leq L_{n}+L_{n-1}+\cdots+L_{m+1} \leq\left(\eta^{n-1}+\eta^{n-2}+\cdots+\eta^{m}\right) L_{1} \\
& =\eta^{m}\left(1+\eta+\cdots+\eta^{n-m-1}\right) L_{1} \leq \eta^{N+1} \frac{1-\eta^{n-m}}{1-\eta} L_{1} \leq \frac{\eta^{N+1}}{1-\eta} L_{1}
\end{aligned}
$$

for $n \geq m>N$, where $N$ is a positive integer. This shows that $S\left(w_{n}-w_{m}\right) \rightarrow 0$ as $n, m \rightarrow \infty$. Thus there exists a system of continuous functions $w_{*}(z)$ in $D^{*}$, such that

$$
S\left(w-w_{*}\right)=C_{\beta}\left[R\left(w_{n}-w_{*}\right), \bar{D}\right]+L_{p_{0}}\left[R \tilde{R}\left(\left|\left(w_{n}-w_{*}\right)_{\bar{z}}\right|+\left|\left(w_{n}-w_{*}\right)_{z}\right|\right), D\right] \rightarrow 0 \text { as } n \rightarrow \infty .
$$

By Condition C, it follows that $w_{*}(z)$ is a solution of Problem B for the system (2.23), i.e. (2.19) for $t \in E$. It is easy to see that the positive constant $\delta$ is independent of $t_{0}\left(0 \leq t_{0}<1\right)$. Hence Problem B for the system (2.19) with $t=t_{0}=0$ is solvable. Correspondingly we can derive that when $t=\delta, 2 \delta, \cdots,[1 / \delta] \delta, 1$, Problem B for (2.19) is solvable. Especially Problem B for (2.19) with $t=1$ and $A(z)=(1-t) F(z, 0,0)$, namely Problem B for the system (1.4) has a solution.

\section{Error Estimates of Approximate Solutions of the Discontinuous Riemann Hilbert Problem for Elliptic Systems of First Order Complex Equations}

In this section, we shall introduce an error estimate of the above approximate solutions.

Theorem 3.1 Under the same conditions as in Theorem 2.3, let $w=w(z)$ be a solution of Problem B for the 
complex system (1.4) satisfying Condition $\mathrm{C}$ in $\bar{D}$, and $w_{n}^{t}=w_{n}(z, t)$ be its approximation as stated in the proof of Theorem 2.3 with $A(z)=(1-t) F(z, 0,0)$. Then we have the following error estimate

$$
\begin{aligned}
S\left(w-w_{n}^{t}\right) & =C_{\beta}\left[R\left(w-w_{n}^{t}\right), \bar{D}\right]+L_{p_{0}}\left[R \tilde{R}\left(\left|\left(w-w_{n}^{t}\right)_{\bar{z}}\right|+\left|\left(w-w_{n}^{t}\right)_{z}\right|\right), \bar{D}\right] \\
& \leq \gamma k\left[\frac{1-\gamma\left|t-t_{0}\right|^{n}}{1-\gamma\left|t-t_{0}\right|}(1-t)+\left(\gamma\left|t-t_{0}\right|\right)^{n}\left(1-t_{0}\right)\right],
\end{aligned}
$$

where $\gamma=M_{3}\left(q_{0}+n k_{0}\right), k=M_{3}\left(k_{2}+k_{5}+k_{6}\right)$ with $M_{3}, q_{0}$ as in (2.28), and $k_{j}(j=2,5,6)$ as in (1.6),(1.7), (1.11) and (1.16).

Proof From (1.4) and (2.24) with $A(z)=(1-t) F(z, 0,0)$, we have

$$
\begin{aligned}
\left(w-w_{n+1}^{t}\right)_{\bar{z}}= & F\left(z, w, w_{z}\right)-t_{0} F\left(z, w_{n+1}^{t}, w_{n+1 z}^{t}\right)-\left(t-t_{0}\right) F\left(z, w_{n}^{t}, w_{n z}^{t}\right)-(1-t) F(z, 0,0) \\
= & (1-t)\left[F\left(z, w, w_{z}\right)-F(z, 0,0)\right]+t_{0}\left[F\left(z, w, w_{z}\right)-F\left(z, w_{n+1}^{t}, w_{n+1 z}^{t}\right)\right] \\
& +\left(t-t_{0}\right) \times\left[F\left(z, w, w_{z}\right)-F\left(z, w_{n}^{t}, w_{n z}^{t}\right)\right] \\
= & t_{0}\left[\tilde{Q}\left(z, w, w_{z}, w_{n+1}^{t}\right)\left(w-w_{n+1}^{t}\right)_{z}+\tilde{A}\left(z, w, w_{n+1}^{t}, w_{n+1 z}^{t}\right)\left(w-w_{n+1}^{t}\right)\right] \\
& +(1-t)\left[F\left(z, w, w_{z}\right)-F(z, 0,0)\right]+\left(t-t_{0}\right)\left[F\left(z, w, w_{z}\right)-F\left(z, w_{n}^{t}, w_{n z}^{t}\right)\right] .
\end{aligned}
$$

It is clear that $w-w_{n+1}^{t}$ satisfies the homogeneous boundary conditions

$$
\begin{aligned}
& \operatorname{Re}\left[\overline{\lambda(z)}\left(w(z)-w_{n+1}^{t}(z)\right)\right]=h(z), \quad z \in \Gamma, \\
& \operatorname{Im}\left[\overline{\lambda\left(a_{j}\right)}\left(w\left(a_{j}\right)-w_{n+1}^{t}\left(a_{j}\right)\right)\right]=0, \quad j \in J_{k}, k=1, \cdots, n .
\end{aligned}
$$

Noting that $\tilde{Q}=\tilde{Q}\left(z, w, w_{z}, w_{n+1}^{t}\right), \tilde{A}=\tilde{A}\left(z, w, w_{n+1}^{t}, w_{n+1 z}^{t}\right)$ satisfy $|\tilde{Q}| \leq q_{0}<1, L_{p_{0}}[\tilde{A}, \bar{D}] \leq k_{0}$, and

$$
\begin{aligned}
& L_{p_{0}}\left[R \tilde{R}\left(F\left(z, w, w_{z}\right)-F(z, 0,0)\right), \bar{D}\right] \\
& \leq q_{0} L_{p_{0}}\left[R \tilde{R} w_{z}, \bar{D}\right]+n k_{0} C[R w, \bar{D}] \\
& \leq\left(q_{0}+n k_{0}\right)\left[L_{p_{0}}\left(R \tilde{R} w_{z}, \bar{D}\right)+C(R w, \bar{D})\right] \\
& \leq\left(q_{0}+n k_{0}\right) S(w), \\
& L_{p_{0}}\left[F\left(z, w, w_{z}\right)-F\left(z, w_{n}^{t}, w_{n z}^{t}\right), \bar{D}\right] \\
& \leq q_{0} L_{p_{0}}\left[R \tilde{R}\left(w-w_{n}^{t}\right)_{z}, \bar{D}\right]+n k_{0} C\left[R\left(w-w_{n}^{t}\right), \bar{D}\right] \\
& \leq\left(q_{0}+n k_{0}\right) \times\left[L_{p_{0}}\left(R \tilde{R}\left(w-w_{n}^{t}\right)_{z}, \bar{D}\right)+C\left(R\left(w-w_{n}^{t}\right), \bar{D}\right)\right] \\
& \leq\left(q_{0}+n k_{0}\right) S\left(w-w_{n}^{t}\right),
\end{aligned}
$$

and then $w-w_{0}^{t}$ is a solution of Problem $B$ for the complex equation

$$
\begin{aligned}
& \left(w-w_{0}^{t}\right)_{\bar{z}}=F\left(z, w, w_{z}\right)-t_{0} F\left(z, w_{0}^{t}, w_{0 z}^{t}\right)-\left(1-t_{0}\right) F(z, 0,0) \\
& =t_{0}\left[F\left(z, w, w_{z}\right)-F\left(z, w_{0}^{t}, w_{0 z}^{t}\right)\right]+\left(1-t_{0}\right)\left[F\left(z, w, w_{z}\right)-F(z, 0,0)\right] \\
& =t_{0} Q\left(w-w_{0}^{t}\right)_{z}+A\left(w-w_{0}^{t}\right)+\left(1-t_{0}\right)\left[F\left(z, w, w_{z}\right)-F(z, 0,0)\right],
\end{aligned}
$$

hence we have

$$
\begin{aligned}
S\left(w-w_{0}^{t}\right) & \leq M_{3}\left(1-t_{0}\right)\left[q_{0} L_{p_{0}}\left(R \tilde{R} w_{z}, \bar{D}\right)+n k_{0} C(R w, \bar{D})\right] \\
& \leq M_{3}\left(q_{0}+n k_{0}\right)\left(1-t_{0}\right) S(w) \leq \gamma\left(1-t_{0}\right) k,
\end{aligned}
$$


in which

$$
S(w) \leq M_{3}\left(k_{2}+k_{5}+k_{6}\right)=k,
$$

where the non-negative constants $M_{3}, k_{2}, k_{5}, k_{6}$ are as stated in (2.28), (1.5), (1.11) and (1.12). Moreover according to the proof of Theorem 2.3, we can derive

$$
\begin{aligned}
S\left(w-w_{n+1}^{t}\right) & \leq M_{3}\left[(1-t)\left(q_{0}+n k_{0}\right) S(w)+\left|t-t_{0}\right|\left(q_{0}+n k_{0}\right) S\left(w-w_{n}^{t}\right)\right] \\
& =M_{3}\left(q_{0}+n k_{0}\right)\left[(1-t) S(w)+\left|t-t_{0}\right| S\left(w-w_{n}^{t}\right)\right] .
\end{aligned}
$$

From (3.6) and (3.7), it follows that

$$
\begin{aligned}
S\left(w-w_{n+1}^{t}\right) \leq & \gamma\left[(1-t) S(w)+\left|t-t_{0}\right| S\left(w-w_{n}^{t}\right)\right] \\
\leq & \gamma(1-t) S(w)\left(1+\gamma\left|t-t_{0}\right|+\gamma^{2}\left|t-t_{0}\right|^{2}+\cdots+\gamma^{n}\left|t-t_{0}\right|^{n}\right) \\
& +\gamma^{n+1}\left|t-t_{0}\right|^{n+1} S\left(w-w_{0}^{t}\right) \\
\leq & \gamma(1-t) S(w) \times \frac{1-\left(\gamma\left|t-t_{0}\right|\right)^{n+1}}{1-\gamma\left|t-t_{0}\right|}+\gamma^{n+1}\left|t-t_{0}\right|^{n+1} S\left(w-w_{0}^{t}\right),
\end{aligned}
$$

where $\gamma=M_{3}\left(q_{0}+n k_{0}\right)$, and $w_{0}^{t}=w\left(z, t_{0}\right)$ is the solution of Problem B for (2.24) with $t=t_{0}$ and $A(z)=\left(1-t_{0}\right) F(z, 0,0)$. Finally, we obtain

$$
\begin{aligned}
S\left(w-w_{n+1}^{t}\right) & \leq \gamma k\left[\frac{1-\gamma\left|t-t_{0}\right|^{n+1}}{1-\gamma\left|t-t_{0}\right|}(1-t)+\gamma k \gamma^{n+1}\left(\left|t-t_{0}\right|\right)^{n+1}\left(1-t_{0}\right)\right] \\
& =\gamma k\left[\frac{1-\gamma\left|t-t_{0}\right|^{n+1}}{1-\gamma\left|t-t_{0}\right|}(1-t)+\left(\gamma\left|t-t_{0}\right|\right)^{n+1}\left(1-t_{0}\right)\right],
\end{aligned}
$$

This shows that (3.1) holds. If the positive constant $\delta$ is small enough, so that when $\left|t-t_{0}\right| \leq \delta, \gamma\left|t-t_{0}\right|<1$, $n$ is sufficiently large and $t$ is close to 1 , then the right hand side becomes very small.

Note: The opinions expressed herein are those of the authors and do not necessarily represent those of the Uniformed Services University of the Health Sciences and the Department of Defense.

\section{References}

[1] Wen, G.C. and Begehr, H. (1990) Boundary Value Problems for Elliptic Equations and Systems. Longman Scientific and Technical Company, Harlow.

[2] Wen, G.C. (1992) Conformal Mappings and Boundary Value Problems, Translations of Mathematics Monographs 106. American Mathematical Society, Providence.

[3] Wen, G.C., Tai, C.W. and Tian, M.Y. (1996) Function Theoretic Methods of Free Boundary Problems and Their Applications to Mechanics. Higher Education Press, Beijing (Chinese).

[4] Wen, G.C. (1986) Linear and Nonlinear Elliptic Complex Equations. Shanghai Scientific and Technical Publishers, Shanghai (Chinese).

[5] Wen, G.C. (1999) Approximate Methods and Numerical Analysis for Elliptic Complex Equations. Gordon and Breach, Amsterdam.

[6] Wen, G.C. (1999 Linear and Nonlinear Parabolic Complex Equations. World Scientific Publishing Co., Singapore City.

[7] Wen, G.C. and Zou, B.T. (2002) Initial-Boundary Value Problems for Nonlinear Parabolic Equations in Higher Dimensional Domains. Science Press, Beijing.

[8] Wen, G.C. (2002) Linear and Quasilinear Complex Equations of Hyperbolic and Mixed Type. Taylor \& Francis, London. http://dx.doi.org/10.4324/9780203166581

[9] Huang, S., Qiao, Y.Y. and Wen, G.C. (2005) Real and Complex Clifford Analysis. Springer Verlag, Heidelberg.

[10] Wen, G.C. (2008) Elliptic, Hyperbolic and Mixed Complex Equations with Parabolic Degeneracy. World Scientific, 
Singapore City.

[11] Wen, G.C., Chen, D.C. and Xu, Z.L. (2008) Nonlinear Complex Analysis and its Applications, Mathematics Monograph Series 12. Science Press, Beijing.

[12] Wen, G.C. (2010) Recent Progress in Theory and Applications of Modern Complex Analysis. Science Press, Beijing. 